\title{
The Error in an Alternating Series
}

\section{Introduction}

Mathematicians have studied the alternating series

$$
\sum_{n=1}^{\infty}(-1)^{n-1} a_{n}=a_{1}-a_{2}+a_{3}-a_{4}+\cdots
$$

since the dawn of analysis. In January of 1714, the great Gottfried Leibniz wrote a letter to Johann Bernoulli in which he explicitly stated his famous criterion for the convergence of (11) as well as the corresponding error estimate [3, p. 33].

Theorem 1. If $a_{n} \geqslant 0$ for $n=0,1, \ldots$ and if the sequence $\left(a_{n}\right)$ decreases monotonically to zero, then the series (11) converges. Let $L$ be its sum. Moreover, let

$$
\begin{aligned}
& S_{n}:=a_{1}-a_{2}+a_{3}-a_{4}+\cdots+(-1)^{n-1} a_{n}, \\
& R_{n}:=L-S_{n}
\end{aligned}
$$

denote its $n^{\text {th }}$ partial sum and remainder, respectively. Then

$$
\left|R_{n}\right| \leqslant a_{n+1},
$$

and $R_{n}$ has the sign $(-1)^{n}$.

It is amazing that the error estimate (8) remained virtually unimproved for almost 250 years! Then, in 1962, Philip Calabrese, a sophomore (!) at the University of Illinois, proved the following significant refinement [2].

Theorem 2. Let $\Delta a_{n}:=a_{n}-a_{n+1}$. If, additionally, the sequence $\left(\Delta a_{n}\right)$ converges monotonically to zero, then

$$
\frac{a_{n+1}}{2}<\left|R_{n}\right|<\frac{a_{n}}{2} .
$$

Calabrese's refined error estimate allowed him to prove the very pretty result that the first partial sum of Leibniz's series

$$
\ln 2=1-\frac{1}{2}+\frac{1}{3}-\frac{1}{4}+\cdots
$$

which approximates $\ln 2$ with four decimal places of accuracy is $S_{10000}$.

Seventeen years later, in 1979, Richard Johnsonbaugh published the following refinement [4] of Calabrese's result. 
Theorem 3. Let $\Delta^{r} a_{n}:=\Delta^{r-1} a_{n}-\Delta^{r-1} a_{n+1}$ for $r=1,2,3, \ldots, k$. If all the sequences $\left(\Delta^{r} a_{n}\right)$ for $r=1,2,3, \ldots, k$ decrease monotonically to zero, then

$$
\frac{a_{n+1}}{2}+\frac{\Delta a_{n+1}}{2^{2}}+\cdots+\frac{\Delta^{k} a_{n+1}}{2^{k+1}}<\left|R_{n}\right|<\frac{a_{n}}{2}-\left\{\frac{\Delta a_{n}}{2^{2}}+\cdots+\frac{\Delta^{k} a_{n}}{2^{k+1}}\right\} .
$$

For example, Johnsonbaugh uses the sharper upper bound estimate (5) with $k=2$ to prove the remarkably precise result that the first partial sum, $S_{n}$, of the series

$$
\frac{\pi}{4}=1-\frac{1}{3}+\frac{1}{5}-\frac{1}{7}+\cdots
$$

that approximates $\frac{\pi}{4}$ with four decimal places of accuracy is $S_{5000}$. Here the precision means that although 4999 terms of the series do not give four decimal places of accuracy, subtracting just one more term, $\frac{1}{9999}$, does give it. This underlines the slowness of the convergence of Leibniz's formula for $\pi$. However, we will show below how to spectacularly accelerate the convergence of this same series.

The lower bound for $\left|R_{n}\right|$ in Johnsonbaugh's theorem (and, in particular, in Calabrese's) had already been found by L. D. Ames in 1901 [1], but the upper bound is new.

Finally, in 1985, Robert M. Young [6] used Cantor's theorem on nested intervals to give an elegant new proof of Calabrese's refinement which makes the error estimates almost intuitive.

It does not seem to have been noticed that Young's method can be adapted to give a new and transparent proof of Johnsonbaugh's refinement.

We elaborate such a proof in this note.

\section{Proof of Johnsonbaugh's Theorem}

Young points out that the crux of Leibniz's original proof of his theorem is this: if the numbers $S_{1}, S_{2}, \ldots$ satisfy the relation

$$
S_{n+1}-S_{n}=(-1)^{n} a_{n+1},
$$

then, since the sequence $a_{n+1}$ decreases to zero, the sequence of closed intervals

$$
\left[S_{2}, S_{1}\right], \quad\left[S_{4}, S_{3}\right], \quad\left[S_{6}, S_{5}\right], \cdots
$$

is nested. Therefore, Cantor's theorem on nested intervals shows that there is a number $L$ common to all these intervals, and that

$$
L=\lim _{n \rightarrow \infty} S_{n},
$$

whereby Leibniz's error estimate is immediate.

To prove Calabrese's theorem, Young defines

$$
T_{n}:=S_{n}+(-1)^{n} \cdot \frac{a_{n+1}}{2} .
$$


Then

$$
T_{n+1}-T_{n}=(-1)^{n} \Delta a_{n+1},
$$

which has the same form as (6). We note that

$$
\left[S_{2 r}, S_{2 r-1}\right] \supseteq\left[T_{2 r}, T_{2 r-1}\right] \quad \text { for } \quad r=1,2, \ldots
$$

Since $\Delta a_{n+1}$ decreases to zero, Cantor's theorem on nested intervals shows that there is a number, $L_{1}=L$, common to all the intervals

$$
\left[T_{2}, T_{1}\right], \quad\left[T_{4}, T_{3}\right], \quad\left[T_{6}, T_{5}\right], \cdots
$$

and that

$$
L=\lim _{n \rightarrow \infty} T_{n}
$$

Now

$$
T_{n}-S_{n}=(-1)^{n} \cdot \frac{a_{n+1}}{2}
$$

and

$$
\begin{aligned}
T_{n-1}-S_{n} & =S_{n-1}+(-1)^{n-1} \cdot \frac{a_{n}}{2}-S_{n} \\
& =(-1)^{n-1} \cdot \frac{a_{n}}{2}-(-1)^{n-1} a_{n}=(-1)^{n} a_{n} .
\end{aligned}
$$

Therefore, if $n$ is even,

$$
\begin{aligned}
& L-S_{n} \geqslant T_{n}-S_{n}=\frac{a_{n+1}}{2}, \\
& L-S_{n} \leqslant T_{n-1}-S_{n}=\frac{a_{n}}{2},
\end{aligned}
$$

which are Calabrese's inequalities. A similar argument holds if $n$ is odd.

To prove Johnsonbaugh's theorem, we have to suitably generalize (7). Let

$$
\begin{array}{cc}
T_{n} & :=S_{n}+(-1)^{n} \cdot \frac{a_{n+1}}{2}, \\
T_{n}^{\prime} & :=T_{n}+(-1)^{n} \cdot \frac{\Delta a_{n+1}}{2^{2}}, \\
T_{n}^{\prime \prime} & :=T_{n}^{\prime}+(-1)^{n} \cdot \frac{\Delta^{2} a_{n+1}}{2^{3}}, \\
\vdots & \quad \vdots \\
T_{n}^{(k)} & :=T_{n}^{(k-1)}+(-1)^{n} \cdot \frac{\Delta^{k} a_{n+1}}{2^{k+1}} .
\end{array}
$$

Now we apply the reasoning we had already applied to $S_{n}$ and $T_{n}$. We note that

$$
\left[S_{2 r}, S_{2 r-1}\right] \supseteq\left[T_{2 r}, T_{2 r-1}\right] \supseteq\left[T_{2 r}^{\prime}, T_{2 r-1}^{\prime}\right] \supseteq \cdots \supseteq\left[T_{2 r}^{(k)}, T_{2 r-1}^{(k)}\right]
$$


for $r=1,2, \ldots$.

Since $\Delta^{k} a_{n+1}$ decreases to zero, Cantor's theorem on nested intervals shows that there is a number, $L_{k}=L$, common to all the intervals

$$
\left[T_{2}^{(k)}, T_{1}^{(k)}\right], \quad\left[T_{4}^{(k)}, T_{3}^{(k)}\right], \quad\left[T_{6}^{(k)}, T_{5}^{(k)}\right], \cdots
$$

and that

$$
L=\lim _{n \rightarrow \infty} T_{n}^{(k)}
$$

Substituting recursively in the definitions of $T_{n}^{(r)}$ for $r=1,2, \ldots$, some simple algebra leads us to the equations

$$
\begin{aligned}
& T_{n}^{(k)}-S_{n}=(-1)^{n}\left(\frac{a_{n+1}}{2}+\frac{\Delta a_{n+1}}{2^{2}}+\cdots+\frac{\Delta^{k} a_{n+1}}{2^{k+1}}\right), \\
& T_{n-1}^{(k)}-S_{n}=(-1)^{n}\left(\frac{a_{n}}{2}-\frac{\Delta a_{n}}{2^{2}}-\cdots-\frac{\Delta^{k} a_{n}}{2^{k+1}}\right) .
\end{aligned}
$$

Therefore, if $n$ is even,

$$
\begin{aligned}
& L-S_{n} \geqslant T_{n}^{(k)}-S_{n}=\frac{a_{n+1}}{2}+\frac{\Delta a_{n+1}}{2^{2}}+\cdots+\frac{\Delta^{k} a_{n+1}}{2^{k+1}}, \\
& L-S_{n} \leqslant T_{n-1}^{(k)}-S_{n}=\frac{a_{n}}{2}-\frac{\Delta a_{n}}{2^{2}}-\cdots-\frac{\Delta^{k} a_{n}}{2^{k+1}},
\end{aligned}
$$

which are Johnsonbaugh's inequalities. A similar argument holds for $n$ odd. This completes the proof.

\section{Euler's transformation}

We saw that Leibniz's series for $\log 2$ and $\frac{\pi}{4}$, while esthetically pleasing, are useless for practical computation because of the slowness of convergence. To rectify this situation, Euler stated the following important transformation formula:

Theorem 4. If all the sequences $\left(\Delta^{r} a_{n}\right)$ for $r=1,2,3, \ldots$ decrease monotonically to zero, then the "Euler transform series"

$$
\frac{a_{1}}{2}+\frac{\Delta a_{1}}{2^{2}}+\frac{\Delta^{2} a_{1}}{2^{3}}+\frac{\Delta^{3} a_{1}}{2^{4}}+\cdots
$$

of the alternating series $a_{1}-a_{2}+a_{3}-+\cdots$ also converges and, indeed, to the same sum, L. If

$$
E_{n}:=\frac{a_{1}}{2}+\frac{\Delta a_{1}}{2^{2}}+\frac{\Delta^{2} a_{1}}{2^{3}}+\frac{\Delta^{3} a_{1}}{2^{4}}+\cdots+\frac{\Delta^{n-1} a_{1}}{2^{n}}
$$

be the $n$-th partial sum, then the error, $r_{n}:=L-E_{n}$, in the approximation $L \approx E_{n}$ satisfies

$$
0<r_{n} \leqslant \frac{\Delta^{n} a_{1}}{2^{n}}
$$

which, shows that $E_{n}$ underestimates $L$. 
Proof. A simple computation shows that that

$$
T_{n+1}^{(k)}-T_{n}^{(k)}=(-1)^{n} \frac{\Delta^{k+1} a_{n+1}}{2^{k+1}} .
$$

By Cantor's theorem the intervals with end-points $T_{n}^{(k)}$ and $T_{n+1}^{(k)}$, in that or opposite order depending on the parity of $n$, close down on $L$, and in particular, $L$ is in all of them. Therefore

$$
\left|L-T_{n}^{(k)}\right|<\frac{\Delta^{k+1} a_{n+1}}{2^{k+1}} .
$$

Taking $n=0$ and then $k=n-1$, we obtain the inequality

$$
\frac{a_{1}}{2}+\frac{\Delta a_{1}}{2^{2}}+\cdots+\frac{\Delta^{n-1} a_{1}}{2^{n}}<L<\frac{a_{1}}{2}+\frac{\Delta a_{1}}{2^{2}}+\cdots+\frac{\Delta^{n-1} a_{1}}{2^{n}}+\frac{\Delta^{n} a_{1}}{2^{n}},
$$

But, the left-hand side is $E_{n}$, and therefore the error estimate (10) is valid.

As an example of Euler's transformation let's compute $\frac{\pi}{4}$ with four decimal digit accuracy from Leibniz's series. We already know that the first partial sum of the Leibniz series that achieves this accuracy is $S_{5000}$. If we compute the differences in the Euler transformation we find that

$$
\Delta^{n} a_{1}=2^{n} \frac{1 \cdot 2 \cdot 3 \cdots n}{1 \cdot 3 \cdot 5 \cdots 2 n+1} .
$$

The error estimate shows that $n$ must satisfy

$$
\frac{1 \cdot 2 \cdot 3 \cdots n}{1 \cdot 3 \cdot 5 \cdots 2 n+1} \leqslant \frac{1}{20000}
$$

and the first value of $n$ which works is $n=13$ with an upper bound for the error given by $2.91 * 10^{-5}$. Therefore, our theorem states that

$$
\begin{aligned}
E_{13} & =\frac{1}{2}\left\{1+\frac{1}{3}+\frac{1 \cdot 2}{3 \cdot 5}+\cdots+\frac{1 \cdot 2 \cdot 3 \cdots 12}{1 \cdot 3 \cdot 5 \cdots 25}\right\} \\
& =\frac{1314078208}{1673196525} \\
& =0.78536991 \ldots
\end{aligned}
$$

approximates $\frac{\pi}{4}$ with an error no larger than $2.91 * 10^{-5}$. In fact the true error is $2.852 *$ $10^{-5}$ which is impresively close to the predicted upper bound for the error. Moreover one only needs 13 summands instead of 5000 to reach the desired accuracy, an extraordinary acceleration of the rate of convergence.

In practice one normally computes a partial sum of the series exactly, and then uses the Euler transform to compute the remainder. For example if we compute $S_{10}$ exactly and apply Euler's transform to the next 11 summands we obtain 0.785398163 which is correct to nine decimal places!

We note that the inequality (12) appears as a problem (without a solution) on p.270 of [5]. 


\section{Acknowledgment}

We thank the referees for suggestions which substantially improved the style and content of this paper. In particular we thank a referee for suggesting the relation of our method of proof to Euler's transformation. Support from the Vicerrectoría de Investigación of the University of Costa Rica is gratefully acknowledged.

\section{References}

[1] L. D. Ames, Evaluation of slowly convergent series, Ann. Math. 3 (1901/02) 185-192.

[2] P. Calabrese, A note on alternating series, Amer. Math. Monthly 69 (1962) 215-217.

[3] G. Ferraro, The Rise and Development of the Theory of Series up to the Early 1820s, Springer, New York, 2008.

[4] R. Johnsonbaugh, Summing an alternating series, Amer. Math. Monthly 86 (1979) 637648.

[5] Konrad Knopp, Theory and Application of Infinite Series, Dover, New York, 1990.

[6] R. M. Young, The error in alternating series, Mathl. Gazette 69 (1985) 120-121. 